\title{
Endoscopic Endonasal Odontoidectomy with Nasopharyngeal Flap Reconstruction
}

\author{
Nyall R. London Jr. ${ }^{1}$ Ahmed Mohyeldin ${ }^{2}$ Ricardo L. Carrau ${ }^{1}$ Daniel M. Prevedello ${ }^{2}$
} ${ }^{1}$ Department of Otolaryngology-Head and Neck Surgery, The Ohio
State University, Columbus, Ohio, United States
2 Department of Neurosurgery, The Ohio State University, Columbus,
Ohio, United States
Address for correspondence Daniel M. Prevedello, MD, Department of Neurological Surgery, Wexner Medical Center at The Ohio State University, N-1049 Doan Hall, 410 West 10th Avenue, Columbus OH 43210, United States (e-mail: daniel.prevedello@osumc.edu).

J Neurol Surg B 2021;82(suppl S1):S12-S13.

\begin{abstract}
Keywords

- basilar invagination

- endoscopic odontoidectomy

- odontoidectomy

Objective This study aimed to demonstrate the nuances in preoperative management, surgical technique, and reconstruction for an endoscopic endonasal odontoidectomy.

Design Assembly of an operative video demonstrating technique for endoscopic endonasal odontoidectomy.

Setting this study is a comprehensive skull base team at a tertiary care center. Participant The patient is a 53-year-old male, with basilar invagination and myelopathy, who underwent cervical fusion, 6 years back, without ventral decompression at an outside hospital. He presented to our clinic with persistent myelopathy and generalized weakness, thus an endoscopic endonasal odontoidectomy for brainstem decompression was recommended.

Main Outcome Measures Preoperative computed tomography (CT) angiography and intraoperative CT navigation demonstrated normal carotid artery anatomic localization. An inverted U-shaped mucosal flap was reflected inferiorly and preserved. The C1 arch was identified and resected with a high speed drill. The resultant diseased soft tissue arising from retropulsion of the odontoid process was then removed and the odontoid process identified. This bone was removed centrally until a thin cap remained. After removal of the cap, the underlying ligamentous tissue was removed until dural pulsations were appreciated and brainstem decompression achieved. Hemostasis was attained and the mucosal flap mobilized into position.
\end{abstract}

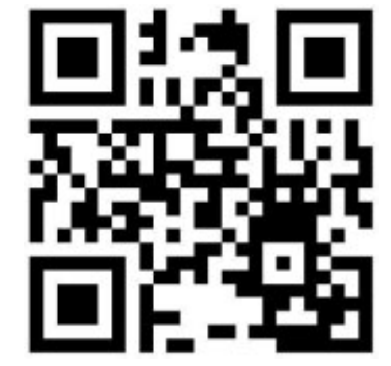

received

January 18, 2019 accepted after revision March 8, 2020

published online

November 23, 2020
DOI https://doi.org/ 10.1055/s-0040-1714408. ISSN 2193-6331.

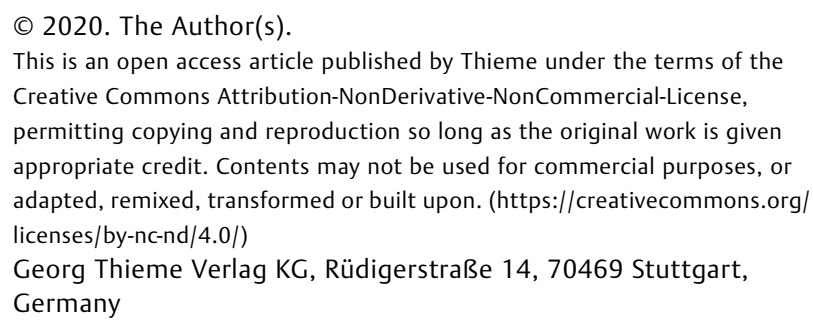


Results Postoperative CT imaging demonstrated resolution of basilar invagination and brainstem decompression (-Fig. 1). The patient improved both in arm dexterity and ambulation after surgery and the reconstruction demonstrated appropriate healing on nasal endoscopy 2 months postoperatively.

Conclusions This operative video demonstrates nuances in endoscopic endonasal odontoidectomy. This case also demonstrates that ventral decompression after longterm cervical fusion can improve myelopathy and that fusion in the setting of bony ventral compression, rather than rheumatoid panus, may not reduce over time with fusion only.

The link to the video can be found at: https://youtu.be/370FFuBA89Y.
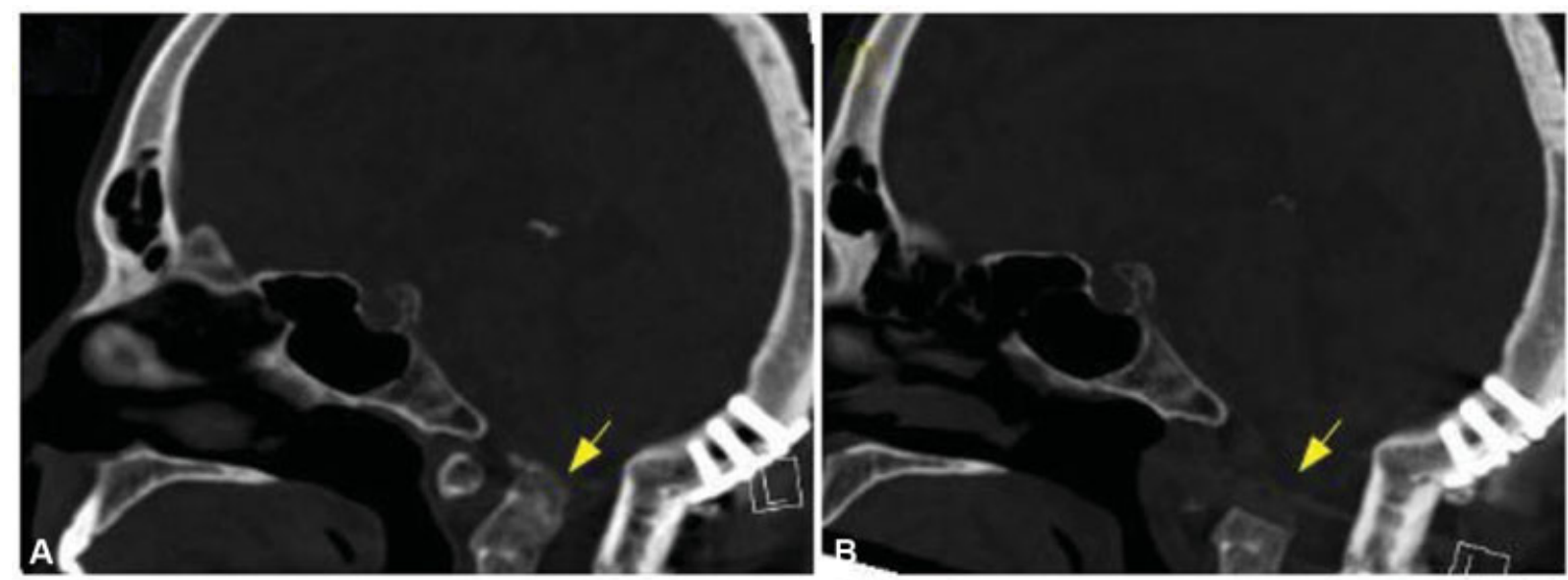

Fig. 1 (A) Preoperative sagittal CT imaging demonstrates retropulsion of the odontoid process and basilar invagination. (B) Postoperative sagittal CT demonstrates removal of the anterior $\mathrm{C} 1$ arch and odontoid process resulting in brainstem decompression. $\mathrm{CT}$, computed tomography.

\section{Conflict of Interest}

R.L.C. is a consultant for Medtronic. D.M.P. is a consultant for Medtronic, Codman, and Stryker. D.M.P. has received honorarium from Leica Microsystems and has a royalty agreement with KLS-Martin. N.R.L. is a consultant for Cooltech Inc. 